QUARTERLY OF APPLIED MATHEMATICS

VOLUME LXIV, NUMBER 3

SEPTEMBER 2006, PAGES 463-482

S $0033-569 X(06) 01026-5$

Article electronically published on June 21, 2006

\title{
MULTIPLICITY OF ASYMMETRIC SOLUTIONS FOR NONLINEAR ELLIPTIC PROBLEMS
}

\author{
BY \\ DAOMIN CAO (Institute of Applied Mathematics, AMSS, Chinese Academy of Sciences, Beijing \\ 100080, People's Republic of China), \\ EZZAT S. NOUSSAIR (School of Mathematics, University of New South Wales, Sydney, NSW 2052, \\ Australia), \\ AND \\ SHUSEN YAN (School of Mathematics, Statistics and Computer Science, The University of New \\ England, Armidale, NSW 2351, Australia)
}

Abstract. In this paper we study the existence of multiple asymmetric positive solutions for the following symmetric problem:

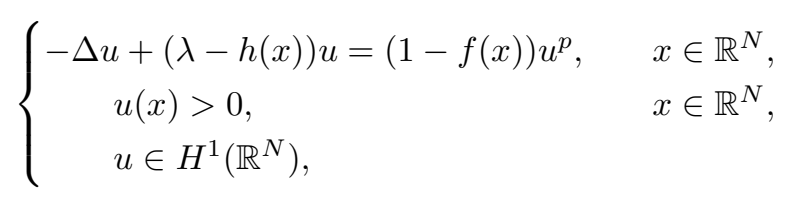

where $\lambda>0$ is a parameter, $h(x)$ and $f(x)$ are nonnegative radially symmetric functions in $L^{\infty}\left(\mathbb{R}^{N}\right), h(x)$ and $f(x)$ have compact support in $\mathbb{R}^{N}, f(x) \leq 1$ for all $x \in \mathbb{R}^{N}$, $1<p<+\infty$ for $N=1,2,1<p<\frac{N+2}{N-2}$ for $N \geq 3$. We prove that for any $k=1,2, \ldots$, if $\lambda$ is large enough the above problem has positive solutions $u_{\lambda}$ concentrating at $k$ distinct points away from the origin as $\lambda$ goes to $\infty$.

Received August 2, 2005.

2000 Mathematics Subject Classification. Primary 35J65, 35B25; Secondary 35Q60.

Key words and phrases. Nonlinear elliptic equation, asymmetric positive solutions, variational method, critical point.

The first author was supported by the Funds of Distinguished Young Scholars of NSFC and Innovation Funds of CAS in China.

The second author was supported by ARC in Australia.

The third author was supported by ARC in Australia.

E-mail address: dmcao@amt.ac.cn

E-mail address: noussair@maths.unsw.edu.au

E-mail address: syan@turing.une.edu.au 
1. Introduction. We are concerned with the existence of asymmetric positive solutions of the following symmetric nonlinear elliptic problem:

$$
\left\{\begin{array}{l}
-\Delta u+(\lambda-h(x)) u=(1-f(x)) u^{p}, \quad \text { in } \mathbb{R}^{N}, \\
u>0, \text { in } \mathbb{R}^{N}, \\
u \in H^{1}\left(\mathbb{R}^{N}\right),
\end{array}\right.
$$

where $h(x), f(x)$ are nonnegative, radially symmetric functions in $L^{\infty}\left(\mathbb{R}^{N}\right), f(x) \leq 1$ for all $x \in \mathbb{R}^{N}, h$ and $f$ have compact support, $\lambda>\|h\|_{L^{\infty}\left(\mathbb{R}^{N}\right)}, 1<p<+\infty$ for $N=1,2$, and $1<p<\frac{N+2}{N-2}$ for $N \geq 3$.

Problem (1.1) has been widely studied (see [5]-[7, 10]-14, [16, 19], 20], 22, 27] and the references therein). In the one-dimensional case problem (1.1) is a model in nonlinear optics arising in the study of the asymmetric guided waves in a symmetric structure consisting of three layers of dielectric materials whose refractive indices depend on the density of the electric field (see for instance [2, 3, 4, 25] when $h(x)=\chi_{[-d, d]}$, $f(x)=\chi_{[-d, d]}$, where $\chi_{[-d, d]}$ stands for the characteristic function of $[-d, d]$, and $d>0$ is a given number). N.N. Akhmediev proved in 2 that a family of asymmetric solutions bifurcates from the branch of symmetric ones at a certain value $\lambda=\lambda_{0}$, yielding the existence of an asymmetric solution for any $\lambda>\lambda_{0}$.

The same phenomenon is studied in the case $N=1$ by Ambrosetti, Arcoya and Gámez for similar problems by the method of perturbation, and the existence of an asymmetric solution was obtained when the internal layer of the medium is thin $(d \rightarrow 0)$ in [3].

When $N=2$, problem (1.1) represents a convenient scalar approximation of a model arising from the study of the propagation of a monochromatic electric field in optical cylindrical waveguides. Such scalar approximations can be found in the engineering and mathematical literature (see [1, 21, 24]). A mathematical description of the propagation of electromagnetic fields in optical cylindrical waveguides having a defocusing or selffocusing dielectric response can be found in papers of O. John and C. Stuart [18] and C. Stuart [26].

A variational technique has been used by Arcoya, Cingolani and Gámez in [4] for $N=1$ and by Cingolani and Gámez for $N \geq 2$ in 13 , to establish the existence of an asymmetric solution for problem (1.1). The main idea in [4, 13] is: First apply the mountain pass lemma to get the existence of a least-energy solution. Then by comparing the energy of the least-energy solution obtained with those of radially symmetric solutions of (1.1), the authors of 4, 13] prove that the least energy solution is asymmetric. It is worthwhile to point out that $h(x) \not \equiv 0$ is crucial in using the mountain pass lemma to obtain the leastenergy solution. In comparing the energy, 4] and [13] used the special form $f(x)=\chi_{B_{d}}$, where $\chi_{B_{d}}$ stands for the characteristic function of $B_{d}=\left\{x \in \mathbb{R}^{N}|| x \mid<d\right\}$. In the case $h(x) \equiv 0$, it is known that problem (1.1) has no least-energy solution if $f(x) \not \equiv 0$. The mountain pass lemma cannot be applied in this case. To deal with a more general radially symmetric function $f(x)$, we need to use new techniques. 
A lot of work has been done for the following problem related to (1.1):

$$
\left\{\begin{aligned}
-\varepsilon^{2} \Delta u+V(x) u=K(x) u^{p} \quad \text { in } \mathbb{R}^{N}, \\
u>0 \text { in } \mathbb{R}^{N}, \\
u \in H^{1}\left(\mathbb{R}^{N}\right) .
\end{aligned}\right.
$$

See [5, 6, 11, 14, 22, 27, for example. Under certain conditions it is proved in 27. that (1.2) has a least-energy solution (ground state) for $\epsilon>0$ small and such a leastenergy solution concentrating at a global minimum of $K(x)$ in the case $V(x)$ is a positive constant. Generalizations have been made for more general nonlinearity by [14. Among other conditions, $V(x)$ and $K(x)$ are assumed to be at least continuous in all the previous work except in [5], where one positive solution was obtained for $\epsilon=1$ when $V(x) \equiv 1$ and $K(x)$ satisfies certain assumptions. In [28, Z.-Q. Wang has considered the following problem:

$$
\left\{\begin{array}{c}
-\Delta u+\lambda V(x) u=u^{p} \quad \text { in } \mathbb{R}^{N}, \\
u>0 \text { in } \mathbb{R}^{N}, \\
u \in H^{1}\left(\mathbb{R}^{N}\right),
\end{array}\right.
$$

where $\lambda>0, V(x)$ is a continuous, radially symmetric function such that $V$ achieves its global infimum (say $V_{0}$ ) on $\{x|| x \mid=1\}$ and $V(x)>V_{0}$ for $1-\sigma<|x|<1+\sigma$, where $\sigma \in(0,1)$. Let $\mathbf{O}(N)$ be the set of all orthogonal transformations in $\mathbb{R}^{N}$ and let $G$ be a given subgroup of $\mathbf{O}(N)$ satisfying certain conditions. By applying the concentration compactness principle to deal with a local minimization problem in a suitable subspace of $H^{1}\left(\mathbb{R}^{N}\right)$, Z.-Q. Wang proved in 28, that (1.3) has a solution which is $G$-invariant for $\lambda$ large and concentrates on $\{x|| x \mid=1\}$ as $\lambda$ goes to $\infty$. For problem (1.2) with $V(x)$ a positive constant, if $K(x)$ satisfies the same conditions of symmetry as $V(x)$ in (1.3) and $K(x)$ achieves its global maximum (say $K_{0}$ ) on $\{x|| x \mid=1\}$ and $K(x)<K_{0}$ for $1-\sigma<|x|<1+\sigma$, where $\sigma \in(0,1)$, then Wang's method in 28] can be applied to obtain a similar result as in [28] for (1.2) when $\varepsilon$ is small enough.

The main purpose of the present paper is to obtain multiplicity of asymmetric solutions for problem (1.1) with more general $f(x)$ including $f(x)=\chi_{B_{d}}$ as a special case. We use a kind of Lyapunov-Schmidt reduction method and make use of the fact that (1.1) is invariant under any subgroup of $\mathbf{O}(N)$. For any integer $k \geq 2$, we obtain many asymmetric solutions concentrating at $k$ points away from the origin even if $h(x) \equiv 0$ for $\lambda$ large (see Theorems 2.1 and 2.2). Location of the limiting positions of the peaks of these solutions is obtained for certain cases for the first time. It is interesting that for $k \geq 2$ the limiting positions are different for the case $h(x) \not \equiv 0$ and $h(x) \equiv 0$ (see Theorem 2.3). It should be pointed out that in the case $h(x) \equiv 0$, no multiplicity of positive solutions was obtained before, not even for the special case $f(x) \equiv \chi_{B_{d}}$; see Remark 2.4 for more details. In this case, 0 is the infimum of $K(x)=1-f(x)$, different from the case that $K(x)$ achieves its global maximum. We would like to point out that we need $\lambda$ to be large, and only for large $\lambda$ do our results improve those in 13 .

Our approach in this paper can be applied to deal with functions $h(x), f(x)$ that are not radially symmetric. Still multiplicity can be obtained for solutions of problem (1.1) 
when $\lambda$ is large (see Remark 2.4). In this case even the existence of one positive solution is not trivial if $h(x) \equiv 0, f(x) \not \equiv 0$ (see [5, 6]).

We would like to point out that the phenomenon that symmetric problems have asymmetric solutions has been studied by many authors for various kind of problems. For example, in [8], Brezis and Nirenberg proved the existence of an asymmetric solution for some nonlinear elliptic PDEs on an annulus. Some other works dealing with asymmetric solutions to radially symmetric semilinear elliptic equations are [11, 15, 17, 20].

2. Main results and remarks. We consider the following nonlinear problem:

$$
\left\{\begin{array}{l}
-\Delta u+(\lambda-h(x)) u=(1-f(x)) u^{p} \quad \text { in } \mathbb{R}^{N}, \\
u>0 \text { in } \mathbb{R}^{N}, \\
u \in H^{1}\left(\mathbb{R}^{N}\right),
\end{array}\right.
$$

where $\lambda>0$ is a parameter, $1<p<\frac{N+2}{N-2}$ if $N \geq 3,1<p<+\infty$ if $N=1,2 . h$ and $f$ are both radially symmetric functions in $L^{\infty}\left(\mathbb{R}^{N}\right)$. We will use the following assumptions:

(h1) $h(x) \geq 0$ in $\mathbb{R}^{N}$ and $h$ has a compact support,

$(f 1) 0 \leq f(x) \leq 1$ in $\mathbb{R}^{N}, f$ has a compact support.

Let $r_{f}=\sup \left\{r \mid \exists\right.$ some $\left.x \in \mathbb{R}^{N},|x|=r, f(x)>0\right\}$.

( $f 2)$ There exist $\theta>0, f_{0}>0$, such that $f(x) \geq f_{0}$ for any $x$ with $r_{f}-\theta<|x|<r_{f}$.

To state our main results, some notation is in order. Let

$$
\|u\|=\left(\int_{\mathbb{R}^{N}}\left(|\nabla u|^{2}+u^{2}\right)\right)^{\frac{1}{2}}
$$

be the usual norm in $H^{1}\left(\mathbb{R}^{N}\right)$, the completion of $C_{0}^{\infty}\left(\mathbb{R}^{N}\right)$ under the norm. Let $U$ be the unique positive solution of

$$
\left\{\begin{array}{l}
-\Delta u+u=u^{p} \text { in } \mathbb{R}^{N}, \\
u>0 \text { in } \mathbb{R}^{N}, \\
u \in H^{1}\left(\mathbb{R}^{N}\right), u(0)=\max _{x \in \mathbb{R}^{N}} u(x) .
\end{array}\right.
$$

It is known from [6] and [19] that $U$ is radially symmetric, strictly decreasing with respect to $|x|$ and

$$
\left\{\begin{array}{l}
U(x) e^{|x|}|x|^{\frac{N-1}{2}} \rightarrow c_{0}>0 \quad \text { as }|x| \rightarrow \infty, \\
\frac{|\nabla U(x)|}{U(x)} \rightarrow 1 \text { as }|x| \rightarrow \infty
\end{array}\right.
$$

Let $\mathbf{O}(N)$ be the set of all orthogonal transformations in $\mathbb{R}^{N}$ and $G$ be a finite subgroup of $\mathbf{O}(N)$ generated by $g$, that is, $G=\left\{g, g^{2}, \ldots, g^{k}=i d\right\}$ for some integer $k \geq 1$.

Theorem 2.1. Suppose (h1), (f1) and (f2) hold and meas $\left\{x \in \mathbb{R}^{N} \mid h(x)>0\right\}>0$. Then there exists a $\lambda_{0}>0$ such that for $\lambda \geq \lambda_{0}$ problem (2.1) has a solution $u_{\lambda}$ satisfying

$$
u_{\lambda}(x)=\lambda^{\frac{1}{p-1}}\left(U\left(\sqrt{\lambda}\left(x-x_{\lambda}\right)\right)+\omega_{\lambda}(\sqrt{\lambda} x)\right),
$$


where

$$
\begin{aligned}
& \omega_{\lambda} \in H^{1}\left(\mathbb{R}^{N}\right), \quad x_{\lambda} \in \mathbb{R}^{N} \text { and } \\
& \left\|\omega_{\lambda}\right\| \rightarrow 0 \text { as } \lambda \rightarrow+\infty, \\
& x_{\lambda} \in \mathbb{R}^{N} \backslash \operatorname{supp} f,\left|x_{\lambda}\right|>r_{f} \text { for } \lambda \text { large. }
\end{aligned}
$$

Theorem 2.2. Suppose (h1), (f1) and (f2) hold and $G=\left\{g, \ldots, g^{k-1}, g^{k}=i d\right\} \subset$ $\mathbf{O}(N), k \geq 2$. Suppose that $G$ has no fixed point in the unit sphere. Then there is a $\lambda_{0}>0$ such that for $\lambda \geq \lambda_{0}$ problem (2.1) has a solution $u_{\lambda}$ satisfying

$$
u_{\lambda}(x)=\lambda^{\frac{1}{p-1}}\left(\alpha_{\lambda} \sum_{i=1}^{k} U\left(\sqrt{\lambda}\left(x-g^{i} x_{\lambda}\right)\right)+\omega_{\lambda}(\sqrt{\lambda} x)\right),
$$

where $\omega_{\lambda}\left(g^{i} x\right)=\omega_{\lambda}(x)$ for any $i=1, \ldots, k$,

$$
\begin{aligned}
& \alpha_{\lambda} \rightarrow 1,\left\|\omega_{\lambda}\right\| \rightarrow 0 \text { as } \lambda \rightarrow+\infty \\
& x_{\lambda} \in \mathbb{R}^{N} \backslash \operatorname{supp} f,\left|x_{\lambda}\right|>r_{f} \text { for } \lambda \text { large. }
\end{aligned}
$$

Theorem 2.3. Let $u_{\lambda}$ be the solutions obtained in Theorems 2.1 and 2.2.

(i) Suppose $k \geq 1, h(x)=0$ if $|x| \geq r_{f} ; h(x) \geq h_{0}>0$ if $r_{f}-\bar{\theta} \leq|x|<r_{f}$ for some $\bar{\theta} \in\left(0, r_{f}\right)$. Then as $\lambda \rightarrow \infty$,

$$
\frac{\sqrt{\lambda}}{\ln \lambda}\left(\left|x_{\lambda}\right|-r_{f}\right) \rightarrow \frac{1}{p-1}
$$

(ii) Suppose $h \equiv 0, G=G_{1} \times G_{2}, G_{1} \subset \mathbf{O}(2)$ and $G_{2} \subset \mathbf{O}(N-2)$ if $N \geq 3$, where $G$ acts canonically on $\mathbb{R}^{2} \times \mathbb{R}^{N-2}$ and $G_{1}=R_{k} \subset \mathbf{O}(2)$ with $R_{k}$ being the subgroup of rotation by an angle equal to integer multiples of $\frac{2 \pi}{k}, G_{2}=\mathbf{O}(N-2)$ if $N \geq 4$, $G_{2}=\{i d,-i d\}$ if $N=3$. Let $x_{\lambda} \rightarrow x$ as $\lambda \rightarrow \infty$. Then

$$
|x|=\frac{(p+1) r_{f}}{p+1-2 \sin \frac{\pi}{k}} .
$$

We end this section with the following remark.

REMARK 2.4. The case that $h(x)$ and $f(x)$ are not radially symmetric functions can be dealt with by the approach used in the next two sections. Suppose $h\left(g^{i} x\right)=h(x)$, $f\left(g^{i} x\right)=f(x)$ for any $i=1, \ldots, k$. Then Theorem 2.1 and Theorem 2.2 are still true. Notice that if $h(x) \equiv 0$, problem (2.1) has no least-energy solution (see [5] for example). It is known that

$$
E(u)=\frac{1}{2} \int_{\mathbb{R}^{N}}\left(|\nabla u|^{2}+\lambda u^{2}\right)-\frac{1}{p+1} \int_{\mathbb{R}^{N}}(1-f(x))|u|^{p+1}
$$

does not satisfy the Palais-Smale condition, and therefore the existence of even one positive solution is not easy to establish (see [5, 6]). By making use of the symmetry of $f(x)$ (not radially symmetric) we can obtain multiple positive solutions even when $f(x) \equiv \chi_{B_{d}}$. Such a result seems to have not been known before.

REMARK 2.5. In Theorem 2.3 we only deal with the case $\operatorname{supp} f=\operatorname{supph}$. It is easy to see that the technique used to prove Theorem 2.3 is still applicable to deal with the case $\operatorname{supp} f \neq \operatorname{supp} h$.

For a similar problem $(f(x) \equiv 0, h(x) \equiv 0)$ in an exterior domain, see [10].

REMARK 2.6. Theorem 2.2 is still true if $G=G_{1} \times G_{2}$ as in (ii) of Theorem 2.3. 
3. Framework and preliminary results. Suppose that $u$ is a solution of problem (2.1). Let $v=\lambda^{-\frac{1}{p-1}} u$. Then $v$ is a solution of the following problem:

$$
\left\{\begin{array}{l}
-\epsilon^{2} \Delta u+\left(1-\epsilon^{2} h(x)\right) u=(1-f(x)) u^{p} \quad \text { in } \mathbb{R}^{N}, \\
u>0 \text { in } \mathbb{R}^{N}, \\
u \in H^{1}\left(\mathbb{R}^{N}\right),
\end{array}\right.
$$

where $\epsilon>0\left(\epsilon=\frac{1}{\sqrt{\lambda}}\right), \epsilon$ small.

We will consider (3.1) instead of (2.1) in the sequel.

Let $I_{\varepsilon}(u)$ be the variational functional associated with (3.1) defined by

$$
I_{\varepsilon}(u)=\frac{1}{2} \int_{\mathbb{R}^{N}}\left(\varepsilon^{2}|\nabla u|^{2}+\left(1-\varepsilon^{2} h(x)\right) u^{2}\right)-\frac{1}{p+1} \int_{\mathbb{R}^{N}}(1-f(x))|u|^{p+1} .
$$

For $z \in \mathbb{R}^{N}$, set $U_{\varepsilon, z}(x)=U\left(\frac{1}{\varepsilon}(x-z)\right)$. For $u, v \in H^{1}\left(\mathbb{R}^{N}\right)$, set $\langle u, v\rangle_{\varepsilon}=$ $\int_{\mathbb{R}^{N}}\left(\varepsilon^{2} \nabla u \nabla v+u v\right)$ and $\|u\|_{\varepsilon}^{2}=\langle u, u\rangle_{\varepsilon}$. For $y=\left(y^{1}, \ldots y^{k}\right) \in \underbrace{\mathbb{R}^{N} \times \cdots \times \mathbb{R}^{N}}_{k}, \delta>0$, define

$$
\begin{gathered}
E_{\varepsilon, y}^{k}=\left\{v \in H^{1}\left(\mathbb{R}^{N}\right) \mid \begin{array}{c}
\left\langle U_{\varepsilon, y^{i}}, v\right\rangle_{\varepsilon}=\left\langle\frac{\partial U_{\varepsilon, y^{i}}}{\partial y_{\ell}^{2}}, v\right\rangle_{\varepsilon}=0, \\
\text { for } i=1, \ldots, k, \ell=1, \ldots, N\}, \\
\Omega_{\varepsilon, \delta}^{k}=\left\{y=\left(y^{1}, \ldots, y^{k}\right)\left|y^{i} \in \mathbb{R}^{N},\right| y^{i}\left|>r_{f}+\delta \varepsilon \ln \frac{1}{\varepsilon},\right| y^{i}-y^{j} \mid>\varepsilon \delta^{-1}\right. \\
\text { for } i, j=1, \ldots, k, i \neq j\} \\
M_{\varepsilon, \delta}^{k}=\left\{(y, \alpha, v)\left|y \in \Omega_{\varepsilon, \delta}^{k}, \alpha=\left(\alpha_{1}, \ldots, \alpha_{k}\right) \in \mathbb{R}^{k},\right| \alpha_{i}-1 \mid<\delta,\right. \\
\text { for } \left.i=1, \ldots, k, v \in E_{\varepsilon, y}^{k},\|v\|_{\varepsilon}<\delta \varepsilon^{N / 2}\right\} .
\end{array}\right.
\end{gathered}
$$

We look for solutions for problem (3.1) of the form $u_{\varepsilon}(x)=\sum_{i=1}^{k} \alpha_{i} U_{\varepsilon, y^{i}}+v$ with $(y, \alpha, v) \in M_{\varepsilon, \delta}^{k}$ for some suitable $\delta>0$. Define

$$
J_{\varepsilon}(y, \alpha, v)=I_{\varepsilon}\left(\sum_{i=1}^{k} \alpha_{i} U_{\varepsilon, y^{i}}+v\right) .
$$

Let us recall a known fact that is important in our argument. It is known that (see [9], 10, 11] and 22]; for earlier work on a similar problem, see [23]) for $\delta>0$ small, $u=\sum_{i=1}^{k} \alpha_{i} U_{\varepsilon, y^{i}}+v$ is a critical point of $I_{\varepsilon}$ in $H^{1}\left(\mathbb{R}^{N}\right)$ if and only if $(y, \alpha, v)$ is a critical point of $J_{\varepsilon}(y, \alpha, v)$ in $M_{\varepsilon, \delta}^{k}$. We notice that $(y, \alpha, v)$ is a critical point of $J_{\varepsilon}(y, \alpha, v)$ if and only if there exist $\xi_{i}, \eta_{\ell}^{i}$ in $\mathbb{R}(i=1, \ldots, k, \ell=1, \ldots, N)$ such that

$$
\begin{aligned}
\frac{\partial J_{\varepsilon}(y, \alpha, v)}{\partial \alpha_{i}} & =0 \\
\frac{\partial J_{\varepsilon}(y, \alpha, v)}{\partial v}(\varphi) & =\sum_{i=1}^{k} \xi_{i}\left\langle U_{\varepsilon, y^{i}}, \varphi\right\rangle_{\varepsilon}+\sum_{i=1}^{k} \sum_{\ell=1}^{N} \eta_{\ell}^{i}\left\langle\frac{\partial U_{\varepsilon, y^{i}}}{\partial y_{\ell}^{i}}, \varphi\right\rangle_{\varepsilon}
\end{aligned}
$$


for every $\varphi \in H^{1}\left(\mathbb{R}^{N}\right)$,

$$
\frac{\partial J_{\varepsilon}(y, \alpha, v)}{\partial y_{\ell}^{i}}=\sum_{m=1}^{N} \eta_{m}^{i}\left\langle\frac{\partial^{2} U_{\varepsilon, y^{i}}}{\partial y_{\ell}^{i} \partial y_{m}^{i}}, v\right\rangle_{\varepsilon}
$$

For problem (3.1) we have the following result from which Theorem 2.1 and Theorem 2.2 can be deduced.

Theorem 3.1. Let $G=\left\{g, g^{2}, \ldots, g^{k}=i d\right\}$ be a finite subgroup of the orthogonal transformation $\mathbf{O}(N)$ in $\mathbb{R}^{N}$ generated by $g$ and such that $G$ has no fixed point in the unit sphere. Suppose (h1), (f1) and (f2) hold and meas $\left\{x \in \mathbb{R}^{N} \mid h(x)>0\right\}>0$ for $k=1$. Then there exists $\varepsilon_{0}>0$ such that for $\varepsilon \in\left(0, \varepsilon_{0}\right]$ problem (3.1) has a solution $u_{\varepsilon}$ of the form

$$
u_{\varepsilon}(x)=\alpha_{\varepsilon} \sum_{i=1}^{k} U_{\varepsilon, y_{\varepsilon}^{i}}+v_{\varepsilon}
$$

with $\alpha_{\varepsilon}>0, y_{\varepsilon}=:\left(y_{\varepsilon}^{1}, \ldots, y_{\varepsilon}^{k}\right)=\left(g z_{\varepsilon}, g^{2} z_{\varepsilon}, \ldots, g^{k-1} z_{\varepsilon}, z_{\varepsilon}\right) \in \underbrace{\mathbb{R}^{N} \times \ldots \mathbb{R}^{N}}_{k}, v_{\varepsilon} \in$ $E_{\varepsilon, y_{\varepsilon}}^{k}, v_{\varepsilon}\left(g^{i} x\right)=v_{\varepsilon}(x)$ for all $x \in \mathbb{R}^{N}, \quad i=1, \ldots, k-1$ satisfying as $\varepsilon \rightarrow 0$,

$$
\begin{aligned}
& \alpha_{\varepsilon} \rightarrow 1, \quad \varepsilon^{-\frac{N}{2}}\left\|v_{\varepsilon}\right\|_{\varepsilon} \rightarrow 0, \\
& \left|z_{\varepsilon}\right|>r_{f}+c_{0} \varepsilon \ln \frac{1}{\varepsilon},
\end{aligned}
$$

for some $c_{0}>0$.

To show Theorem 3.1. some preliminary results have to be established first. The following result will play an important role in our proof of Theorem 3.1.

Proposition 3.2. For any given positive $\delta$ small enough, there exists an $\varepsilon_{0}>0$, such that for $\varepsilon \in\left(0, \varepsilon_{0}\right]$ there is a unique $C^{1}$-map: $y=\left(y^{1}, \ldots, y^{k}\right) \in \Omega_{\varepsilon, \delta}^{k} \mapsto\left(\alpha_{\varepsilon, y}, v_{\varepsilon, y}\right) \in$ $[1-\delta, 1+\delta]^{k} \times E_{\varepsilon, y}^{k},\left(y, \alpha_{\varepsilon, y}, v_{\varepsilon, y}\right)$ satisfies (3.4) and (3.5) for some $\xi_{i}, \eta_{\ell}^{i}$ for $i=1, \ldots, k$, $\ell=1, \ldots, N$. Furthermore, letting $\alpha_{\varepsilon, y}=\left(\alpha_{\varepsilon, y, 1}, \ldots, \alpha_{\varepsilon, y, k}\right)$ we have

$$
\begin{aligned}
\varepsilon^{\frac{N}{2}} \sum_{i=1}^{k}\left|\alpha_{\varepsilon, y, i}-1\right|+\left\|v_{\varepsilon, y}\right\|_{\varepsilon}= & O\left(\varepsilon^{\frac{N}{2}} \sum_{i=1}^{k} e^{-(1+p-\sigma)\left(\left|y^{i}\right|-r_{f}\right) / \varepsilon}\right) \\
& +O\left(\varepsilon^{\frac{N}{2}} \sum_{i<j}^{k} e^{-q\left|y^{i}-y^{j}\right| / \varepsilon}\right) \\
& +O\left(\varepsilon^{2}\left(\sum_{i=1}^{k} \int_{\mathbb{R}^{N}} h(x) U_{\varepsilon, y^{i}}^{2}\right)^{1 / 2}\right)
\end{aligned}
$$

for any $\sigma>0$, where $q>\frac{1}{2}$.

Proof. Although the idea of our proof of Proposition 3.2 is the same as in 9], 11] and [22], the estimates involved here are quite different and we give a sketch here. 
For $\omega_{i}=\left(\beta^{i}, v_{i}\right) \in \mathbb{R}^{k} \times E_{\varepsilon, y}^{k}, i=1,2$, define

$$
\left(\omega_{1}, \omega_{2}\right)_{\varepsilon}=\varepsilon^{N} \sum_{\ell=1}^{N} \beta_{\ell}^{1} \beta_{\ell}^{2}+\left\langle v_{1}, v_{2}\right\rangle_{\varepsilon} .
$$

Let $[\omega]_{\varepsilon}=\sqrt{(\omega, \omega)_{\varepsilon}} \cdot \mathbb{R}^{k} \times E_{\varepsilon, y}^{k}$ is a linear space with scalar product defined by (3.9). Set $\alpha_{0}=(1, \ldots, 1) \in \mathbb{R}^{k}, \beta=\alpha-\alpha_{0}, \omega=(\beta, v)$ and $J_{\varepsilon}^{*}(y, \omega)=J_{\varepsilon}(y, \alpha, v)$.

Expanding $J_{\varepsilon}^{*}(y, \omega)$ at $\omega_{0}=(0,0)$ we obtain

$$
J_{\varepsilon}(y, \alpha, v)=J_{\varepsilon}\left(y, \alpha_{0}, 0\right)+f_{\varepsilon, y}(\omega)+Q_{\varepsilon, y}(\omega)+R_{\varepsilon, y}(\omega),
$$

where $f_{\varepsilon, y}(\omega)$ is the linear part, $Q_{\varepsilon, y}(\omega)$ is the quadratic part and $R_{\varepsilon, y}(\omega)$ is the remainder of higher order,

$$
\begin{gathered}
f_{\varepsilon, y}(\omega)=-\varepsilon^{2} \int_{\mathbb{R}^{N}} h(x) v \sum_{i=1}^{k} U_{\varepsilon, y^{i}}-\int_{\mathbb{R}^{N}}(1-f(x)) v\left(\sum_{i=1}^{k} U_{\varepsilon, y^{i}}\right)^{p} \\
+\sum_{i=1}^{k} \beta_{i}\left(\int_{\mathbb{R}^{N}}\left(\varepsilon^{2} \nabla U_{\varepsilon, y^{i}} \nabla\left(\sum_{j=1}^{k} U_{\varepsilon, y^{j}}\right)+\left(1-\varepsilon^{2} h(x)\right) U_{\varepsilon, y^{i}} \sum_{j=1}^{k} U_{\varepsilon, y^{j}}\right)\right. \\
\left.-\int_{\mathbb{R}^{N}}(1-f(x)) U_{\varepsilon, y^{i}}\left(\sum_{j=1}^{k} U_{\varepsilon, y^{j}}\right)^{p}\right), \\
Q_{\varepsilon, y}(\omega)=\frac{1}{2} Q_{\varepsilon, y}^{(1)}(v)+\frac{1}{2} Q_{\varepsilon, y}^{(2)}(\beta)+\frac{1}{2} Q_{\varepsilon, y}^{(3)}(\omega),
\end{gathered}
$$

with $Q_{\varepsilon, y}^{(i)}(i=1,2,3)$ being given by the following:

$$
\begin{gathered}
Q_{\varepsilon, y}^{(1)}(v)=\int_{\mathbb{R}^{N}}\left(\varepsilon^{2}|\nabla v|^{2}+\left(1-\varepsilon^{2} h(x)\right) v^{2}\right)-p \int_{\mathbb{R}^{N}}(1-f(x))\left(\sum_{i=1}^{k} U_{\varepsilon, y^{i}}\right)^{p-1} v^{2}, \\
Q_{\varepsilon, y}^{(2)}(\beta)=\sum_{i, j=1}^{k} \beta_{i} \beta_{j} \int_{\mathbb{R}^{N}}\left(\varepsilon^{2} \nabla U_{\varepsilon, y^{i}} \nabla U_{\varepsilon, y^{j}}+\left(1-\varepsilon^{2} h(x)\right) U_{\varepsilon, y^{i}} U_{\varepsilon, y^{j}}\right. \\
\left.-p(1-f(x))\left(\sum_{m=1}^{k} U_{\varepsilon, y^{m}}\right)^{p-1} U_{\varepsilon, y^{i}} U_{\varepsilon, y^{j}}\right), \\
Q_{\varepsilon, y}^{(3)}(\omega)=-\sum_{i=1}^{k} \beta_{i}\left(\varepsilon^{2} \int_{\mathbb{R}^{N}} h(x) U_{\varepsilon, y^{i}} v+p \int_{\mathbb{R}^{N}}(1-f(x))\left(\sum_{j=1}^{k} U_{\varepsilon, y^{j}}\right)^{p-1} U_{\varepsilon, y^{i}} v\right), \\
\begin{cases}R_{\varepsilon, y}(\omega) & =O\left([\omega]_{\varepsilon}^{p_{*}}\right), \\
R_{\varepsilon, y}^{\prime}(\omega) & =O\left([\omega]_{\varepsilon}^{p_{*}-1}\right), \\
R_{\varepsilon, y}^{\prime \prime}(\omega) & =O\left([\omega]_{\varepsilon}^{p_{*}-2}\right),\end{cases}
\end{gathered}
$$

where $p_{*}=\min \{p+1,3\}>2$. in (3.16) and hereafter we will use $O(t)$ to denote all the quantities $C(t)$ such that $\frac{C(t)}{t}$ is bounded for $t>0$ small.

It follows from the fact that $f_{\varepsilon, y}$ is a continuous form over $\mathbb{R}^{k} \times E_{\varepsilon, y}^{k}$ that there exists a unique element $\widehat{f}_{\varepsilon, y} \in \mathbb{R}^{k} \times E_{\varepsilon, y}^{k}$ satisfying

$$
f_{\varepsilon, y}(\omega)=\left(\widehat{f}_{\varepsilon, y}, \omega\right)_{\varepsilon} \quad \text { for any } \omega \in \mathbb{R}^{k} \times E_{\varepsilon, y}^{k} .
$$


In the same way, since $Q_{\varepsilon, y}$ is a continuous quadratic form over $\mathbb{R}^{k} \times E_{\varepsilon, y}^{k}$, there exists a continuous linear operator $A_{\varepsilon, y}(\omega)$ from $\mathbb{R}^{k} \times E_{\varepsilon, y}^{k}$ onto itself such that $Q_{\varepsilon, y}(\omega)=$ $\left(A_{\varepsilon, y} \omega, \omega\right)_{\varepsilon}$ for each $\omega \in \mathbb{R}^{k} \times E_{\varepsilon, y}^{k}$ and

$$
\left(A_{\varepsilon, y} \omega, \omega\right)_{\varepsilon}=\frac{1}{2} Q_{\varepsilon, y}^{(1)}(v)+\frac{1}{2} Q_{\varepsilon, y}^{(2)}(\beta)+\frac{1}{2} Q_{\varepsilon, y}^{(3)}(\omega) .
$$

We will show that for $\varepsilon>0$ small, $A_{\varepsilon, y}$ has a bounded inverse. To do this we analyze $Q_{\varepsilon, y}^{(1)}, Q_{\varepsilon, y}^{(2)}$ and $Q_{\varepsilon, y}^{(3)}$ respectively.

By Lemma 2.2 in [22, there is a $\rho>0$ independent of $y$ and $\varepsilon$ such that

$$
Q_{\varepsilon, y}^{(1)}(v) \geq \rho\|v\|_{\varepsilon}^{2} \text { for all } v \in E_{\varepsilon, y}^{k} .
$$

On the other hand, there exists a constant $C_{N}>0$ such that

$$
\begin{aligned}
Q_{\varepsilon, y}^{(2)}(\beta)= & \sum_{i=1}^{k} \beta_{i}^{2}\left(\int_{\mathbb{R}^{N}}\left(\varepsilon^{2}\left|\nabla U_{\varepsilon, y^{i}}\right|+U_{\varepsilon, y^{i}}^{2}\right)-p \int_{\mathbb{R}^{N}} U_{\varepsilon, y^{i}}^{p+1}+O\left(\varepsilon^{N+2}\right)\right) \\
& +o(1) \sum_{\substack{i, j=1 \\
i \neq j}}\left|\beta_{i}\right|\left|\beta_{j}\right| \varepsilon^{N} \\
\leq & -C_{N} \sum_{i=1}^{k} \beta_{i}^{2} \varepsilon^{N}=-C_{N}|\beta|^{2} \varepsilon^{N} .
\end{aligned}
$$

Here and hereafter, we will use $o(1)$ to denote various quantities that go to zero as $\varepsilon \rightarrow 0+$.

Similarly we have

$$
Q_{\varepsilon, y}^{(3)}(\omega)=o(1)[\omega]_{\varepsilon}^{2} .
$$

From (3.20) and (3.21), we conclude that there exists a unique linear operator $B_{\varepsilon, y}$ from $\mathbb{R}^{k} \times E_{\varepsilon, y}^{k}$ onto itself such that $\left(B_{\varepsilon, y} \omega, \omega\right)_{\varepsilon}=\frac{1}{2} Q_{\varepsilon, y}^{(1)}(v)+\frac{1}{2} Q_{\varepsilon, y}^{(2)}(\beta)$. In view of (3.19) and (3.20), $B_{\varepsilon, y}$ is invertible and $\left\|B_{\varepsilon, y}^{-1}\right\| \leq C^{*}$ for some $C^{*}>0$ independent of $y$ and $\varepsilon$. From (3.21) we obtain $\left\|A_{\varepsilon, y}-B_{\varepsilon, y}\right\|=\frac{1}{2}\left\|Q_{\varepsilon, y}^{(3)}\right\|=o(1)$. Hence for $\varepsilon$ small, $A_{\varepsilon, y}$ is invertible and $\left\|A_{\varepsilon, y}^{-1}\right\| \leq 2 C^{*}$. Now following the argument in [9], 11] (or in [23]) we have

$$
\left.\frac{\partial J^{*}(y, \omega)}{\partial \omega}\right|_{\mathbb{R}^{k} \times E_{\varepsilon, y}^{k}}=\widehat{f}_{\varepsilon, y}+2 A_{\varepsilon, y}+R_{\varepsilon, y}^{\prime}(\omega) .
$$

There exists an equivalence between the existence of $\omega=(\beta, v)$ such that (3.4) and (3.5) hold for $\left(y, \beta+\alpha_{0}, v\right)$ and

$$
\widehat{f}_{\varepsilon, y}+2 A_{\varepsilon, y}+R_{\varepsilon, y}^{\prime}(\omega)=0
$$

As in [23], by the implicit function theorem we get $\varepsilon_{0}>0, \delta_{0}>0$ and a $C^{1}$-map $\omega_{\varepsilon}=\left(\beta_{\varepsilon}, v_{\varepsilon}\right): y \in \Omega_{\varepsilon, \delta}^{k} \mapsto \mathbb{R}^{k} \times E_{\varepsilon, y}^{k}$ for $\varepsilon \in\left(0, \varepsilon_{0}\right], \delta \in\left(0, \delta_{0}\right]$ satisfying (3.22) and

$$
\left[\omega_{\varepsilon}\right]_{\varepsilon} \leq C\left[\widehat{f}_{\varepsilon, y}\right]_{\varepsilon} .
$$


To obtain estimate (3.8) we need to get the estimate of $\left[\widehat{f}_{\varepsilon, y}\right]_{\varepsilon}$. To this end, we need to estimate each integral in (3.11). Using (2.3), we have for any fixed $\sigma>0$,

$$
\begin{aligned}
& \int_{\mathbb{R}^{N}}(1-f(x)) v\left(\sum_{i=1}^{k} U_{\varepsilon, y^{i}}\right)^{p}=\int_{\mathbb{R}^{N}}(1-f(x)) v \sum_{i=1}^{k} U_{\varepsilon, y^{i}}^{p}+O\left(\sum_{i<j}^{k} \int_{\mathbb{R}^{N}} U_{\varepsilon, y^{i}}^{p / 2} U_{\varepsilon, y^{j}}^{p / 2}|v|\right) \\
& =-\int_{\mathbb{R}^{N}} f(x) v \sum_{i=1}^{k} U_{\varepsilon, y^{i}}^{p}+O\left(\varepsilon^{\frac{N}{2}} \sum_{\substack{i, j=1 \\
i \neq j}}^{k} e^{-(p-\sigma)\left|y^{i}-y^{j}\right| /(2 \varepsilon)}\right)\|v\|_{\varepsilon} \\
& =O\left(\varepsilon^{\frac{N}{2}}\left(\sum_{i=1}^{k} e^{-(p-\sigma)\left(\left|y^{i}\right|-r_{f}\right) / \varepsilon}+\sum_{\substack{i, j=1 \\
i \neq j}}^{k} e^{-(p-\sigma)\left|y^{i}-y^{j}\right| /(2 \varepsilon)}\right)\right)\|v\|_{\varepsilon}, \\
& \left|\int_{\mathbb{R}^{N}} h(x) v \sum_{i=1}^{k} U_{\varepsilon, y^{i}}\right| \leq\left(\sum_{i=1}^{k} \int_{\mathbb{R}^{N}} h^{2}(x) U_{\varepsilon, y^{i}}^{2}\right)^{\frac{1}{2}}\|v\|_{\varepsilon} \\
& =O\left(\left(\sum_{i=1}^{k} \int_{\mathbb{R}^{N}} h(x) U_{\varepsilon, y^{i}}^{2}\right)^{\frac{1}{2}}\right)\|v\|_{\varepsilon},
\end{aligned}
$$

and

$$
\begin{aligned}
& \int_{\mathbb{R}^{N}}\left(\varepsilon^{2} \nabla U_{\varepsilon, y^{i}} \nabla\left(\sum_{j=1}^{k} U_{\varepsilon, y^{j}}\right)+\left(1-\varepsilon^{2} h(x)\right) U_{\varepsilon, y^{i}} \sum_{j=1}^{k} U_{\varepsilon, y^{j}}\right) \\
& -\int_{\mathbb{R}^{N}}(1-f(x)) U_{\varepsilon, y^{i}}\left(\sum_{j=1}^{k} U_{\varepsilon, y^{j}}\right)^{p} \\
& =\sum_{j=1}^{k} \int_{\mathbb{R}^{N}} U_{\varepsilon, y^{i}}^{p} U_{\varepsilon, y^{j}}-\varepsilon^{2} \int_{\mathbb{R}^{N}} h(x) U_{\varepsilon, y^{i}} \sum_{j=1}^{k} U_{\varepsilon, y^{j}}-\int_{\mathbb{R}^{N}}(1-f(x)) U_{\varepsilon, y^{i}}^{p+1} \\
& +O\left(\sum_{\substack{i, j=1 \\
i \neq j}}^{k} \int_{\mathbb{R}^{N}} U_{\varepsilon, y^{i}}^{p} U_{\varepsilon, y^{j}}\right) \\
& =-\varepsilon^{2} \int_{\mathbb{R}^{N}} h(x) U_{\varepsilon, y^{i}}^{2}+O\left(\varepsilon^{N} \sum_{\substack{i, j=1 \\
i \neq j}}^{k} e^{-(1-\sigma)\left|y^{i}-y^{j}\right| / \varepsilon}\right) \\
& +O\left(\varepsilon^{N} \sum_{i=1}^{k} e^{-(p+1-\sigma)\left(\left|y^{i}\right|-r_{f}\right) / \varepsilon}\right) .
\end{aligned}
$$


Using (3.24), (3.26) and (3.25) in (3.11) we derive for some $q>\frac{1}{2}$, any $\sigma>0$ and $\varepsilon>0$ small,

$$
\begin{aligned}
{\left[\omega_{\varepsilon}\right]_{\varepsilon} } & =O\left(\varepsilon^{\frac{N}{2}} \sum_{\substack{i, j=1 \\
i \neq j}}^{k} e^{-q\left|y^{i}-y^{j}\right| / \varepsilon}\right) \\
& +O\left(\varepsilon^{\frac{N}{2}} \sum_{i=1}^{k} e^{-(p+1-\sigma)\left(\left|y^{i}\right|-r_{f}\right) / \varepsilon}\right)+O\left(\varepsilon^{2}\left(\int_{\mathbb{R}^{N}} h(x) \sum_{i=1}^{k} U_{\varepsilon, y^{i}}^{2}\right)^{\frac{1}{2}}\right),
\end{aligned}
$$

which deduces (3.8) and completes our proof.

4. Proof of the main results. In this section, we give proofs of Theorem 2.1 and Theorem 2.2 To do this, we need only show Theorem 3.1

Denote, for $\delta>0, R>r_{f}+\delta \varepsilon \ln \frac{1}{\varepsilon}$, the annulus in $\mathbb{R}^{N}$ by

$$
B_{\delta, R}=\left\{z \in \mathbb{R}^{N}\left|r_{f}+\delta \varepsilon \ln \frac{1}{\varepsilon}<\right| z \mid<R\right\}
$$

Let $G=\left\{g, g^{2}, \ldots, g^{k}=i d\right\} \subset \mathbf{O}(N)$ and set

$$
D_{\delta, R}=\left\{y=\left(g z, g^{2} z, \ldots, g^{k-1} z, z\right) \mid z \in B_{\delta, R}\right\}
$$

We will establish the existence of the critical point of $J_{\varepsilon}\left(y, \alpha_{\varepsilon, y}, v_{\varepsilon, y}\right)$ in $D_{\delta, R}$ via solving the following minimization problem:

$$
m_{\varepsilon, \delta, R}=\inf \left\{J_{\varepsilon}\left(y, \alpha_{\varepsilon, y}, v_{\varepsilon, y}\right) \mid y \in \bar{D}_{\delta, R}\right\}
$$

for $\delta>0$ small and $R>0$ large, where $\left(\alpha_{\varepsilon, y}, v_{\varepsilon, y}\right)$ is obtained as in Proposition 3.2 for $y \in \bar{D}_{\delta, R}$.

First we prove that $m_{\varepsilon, \delta, R}$ can be achieved by some interior point in $D_{\delta, R}$.

Lemma 4.1. There exist $\delta>0, R>r_{f}+\delta \varepsilon \ln \frac{1}{\varepsilon}$ such that $m_{\varepsilon, \delta, R}$ is achieved by some $y_{\varepsilon} \in D_{\delta, R}$ if $\varepsilon>0$ is small enough.

Proof. For any $z \in B_{\delta, R}$, denote $|z|=r$. Suppose $y=\left(g z, g^{2} z, \ldots, g^{k-1} z, z\right)$. We have for $i \neq j,\left|y^{i}-y^{j}\right|=\left|g^{i} z-g^{j} z\right|=\theta_{i j} r$, where $\theta_{i j} \in\left(\theta_{0}, 2\right]$ with $\theta_{0}>0$ depending on $k$ and $N$ only. $\theta_{i j}=2$ if $k=2$, and $\theta_{i j}<2$ if $k \geq 3$.

Next we show that for some $1 \gg \delta>0, R \gg 1$,

$$
m_{\varepsilon, \delta, R}<\min \left\{J_{\varepsilon}\left(y, \alpha_{\varepsilon, y}, v_{\varepsilon, y}\right) \mid y \in \partial D_{\delta, R}\right\} .
$$


To this end, we need to estimate $J_{\varepsilon}\left(y, \alpha_{\varepsilon, y}, v_{\varepsilon, y}\right)$ for $y \in \partial D_{\delta, R}$. From the proof of Proposition 3.2 we have

$$
\begin{aligned}
J_{\varepsilon}\left(y, \alpha_{\varepsilon, y}, v_{\varepsilon, y}\right)= & J_{\varepsilon}\left(y, \alpha_{0}, 0\right)+O\left(\left[\omega_{\varepsilon}\right]_{\varepsilon}^{2}\right) \\
= & J_{\varepsilon}\left(y, \alpha_{0}, 0\right)+O\left(\varepsilon^{N} \sum_{\substack{i, j=1 \\
i \neq j}}^{k} e^{-2 q \theta_{i j} r / \varepsilon}\right) \\
& +O\left(\varepsilon^{4}\left(\int_{\mathbb{R}^{N}} h(x) \sum_{i=1}^{k} U_{\varepsilon, y^{i}}^{2}\right)\right) \\
& +O\left(\varepsilon^{N} e^{\left.-(2 p-2 \sigma)\left(r-r_{f}\right) / \varepsilon\right)}\right) .
\end{aligned}
$$

Denote $A=\frac{1}{2} \int_{\mathbb{R}^{N}}\left(|\nabla U|^{2}+U^{2}\right)-\frac{1}{p+1} \int_{\mathbb{R}^{N}} U^{p+1}$. Then

$$
\begin{aligned}
J_{\varepsilon}\left(y, \alpha_{0}, 0\right)= & \frac{1}{2} \int_{\mathbb{R}^{N}}\left(\varepsilon^{2}\left|\nabla\left(\sum_{i=1}^{k} U_{\varepsilon, y^{i}}\right)\right|^{2}+\left(1-\varepsilon^{2} h(x)\right)\left(\sum_{i=1}^{k} U_{\varepsilon, y^{i}}\right)^{2}\right) \\
& -\frac{1}{p+1} \int_{\mathbb{R}^{N}}(1-f(x))\left(\sum_{i=1}^{k} U_{\varepsilon, y^{i}}\right)^{p+1} \\
= & \sum_{i=1}^{k}\left(\frac{1}{2} \int_{\mathbb{R}^{N}}\left(\varepsilon^{2}\left|\nabla U_{\varepsilon, y^{i}}\right|^{2}+U_{\varepsilon, y^{i}}^{2}\right)-\frac{1}{p+1} \int_{\mathbb{R}^{N}} U_{\varepsilon, y^{i}}^{p+1}\right) \\
& +\frac{1}{2} \sum_{i, j=1}^{k} \int_{\mathbb{R}^{N}}\left(\varepsilon^{2} \nabla U_{\varepsilon, y^{i}} \nabla U_{\varepsilon, y^{j}}+U_{\varepsilon, y^{i}} U_{\varepsilon, y^{j}}\right) \\
& -\frac{\varepsilon^{2}}{2} \int_{\mathbb{R}^{N}} h(x)\left(\sum_{i=1}^{k} U_{\varepsilon, y^{i}}\right)^{2} \\
& -\frac{1}{p+1} \int_{\mathbb{R}^{N}}\left((1-f(x))\left(\sum_{i=1}^{k} U_{\varepsilon, y^{i}}\right)^{p+1}-\sum_{i=1}^{k} U_{\varepsilon, y^{i}}^{p+1}\right) \\
= & k \varepsilon^{N} A-\frac{\varepsilon^{2}}{2} \int_{\mathbb{R}^{N}} h(x)\left(\sum_{i=1}^{k} U_{\varepsilon, y^{i}}\right)^{2}+\frac{1}{2} \sum_{i, j=1}^{k} \int_{\mathbb{R}^{N}} U_{\varepsilon, y^{i}}^{p} U_{\varepsilon, y^{j}} \\
& -\frac{1}{p+1} \int_{\mathbb{R}^{N}}\left((1-f(x))\left(\sum_{i=1}^{k} U_{\varepsilon, y^{i}}\right)^{p+1}-\sum_{i=1}^{k} U_{\varepsilon, y^{i}}^{p+1}\right) .
\end{aligned}
$$


On the other hand, we have

$$
\begin{aligned}
\int_{\mathbb{R}^{N}}( & \left.(1-f(x))\left(\sum_{i=1}^{k} U_{\varepsilon, y^{i}}\right)^{p+1}-\sum_{i=1}^{k} U_{\varepsilon, y^{i}}^{p+1}\right) \\
= & \int_{\mathbb{R}^{N}}(1-f(x))\left(\sum_{i=1}^{k} U_{\varepsilon, y^{i}}^{p+1}+(p+1) \sum_{\substack{i, j=1 \\
i \neq j}}^{k} U_{\varepsilon, y^{i}}^{p} U_{\varepsilon, y^{j}}\right)-\int_{\mathbb{R}^{N}} \sum_{i=1}^{k} U_{\varepsilon, y^{i}}^{p+1} \\
& +O\left(\sum_{\substack{i, j=1 \\
i \neq j}}^{k} \int_{\mathbb{R}^{N}} U_{\varepsilon, y^{i}}^{\frac{p+1}{2}} U_{\varepsilon, y^{j}}^{\frac{p+1}{2}}\right) \\
= & \int_{\mathbb{R}^{N}} f(x) \sum_{i=1}^{k} U_{\varepsilon, y^{i}}^{p+1}-(p+1) \int_{\mathbb{R}^{N}} f(x) \sum_{\substack{i, j=1 \\
i \neq j}}^{k} U_{\varepsilon, y^{i}}^{p} U_{\varepsilon, y^{j}} \\
& +\quad(p+1) \int_{\mathbb{R}^{N}} \sum_{\substack{i, j=1 \\
i \neq j}}^{k} U_{\varepsilon, y^{i}}^{p} U_{\varepsilon, y^{j}}+O\left(\sum^{N} \sum_{\substack{i, j=1 \\
i \neq j}}^{k} e^{-\left(1+\sigma_{0}\right) \theta_{i j} r / \varepsilon}\right)
\end{aligned}
$$

for some $\sigma_{0}>0$.

Substituting (4.4), (4.5) into (4.3), we obtain

$$
\begin{aligned}
J_{\varepsilon}\left(y, \alpha_{\varepsilon, y}, v_{\varepsilon, y}\right)=k \varepsilon^{N} A-\frac{\varepsilon^{2}}{2} \int_{\mathbb{R}^{N}} h(x)\left(\sum_{i=1}^{k} U_{\varepsilon, y^{i}}\right)^{2} \\
\quad-\frac{1}{2} \sum_{\substack{i, j=1 \\
i \neq j}}^{k} \int_{\mathbb{R}^{N}} U_{\varepsilon, y^{i}}^{p} U_{\varepsilon, y^{j}}+\frac{1}{p+1} \sum_{i=1}^{k} \int_{\mathbb{R}^{N}} f(x) U_{\varepsilon, y^{i}}^{p+1} \\
+\sum_{\substack{i, j=1 \\
i \neq j}}^{k} \int_{\mathbb{R}^{N}} f(x) U_{\varepsilon, y^{i}}^{p} U_{\varepsilon, y^{j}}+O\left(\varepsilon^{4} \sum_{i=1}^{k} \int_{\mathbb{R}^{N}} h(x) U_{\varepsilon, y^{i}}^{2}\right) \\
+O\left(\varepsilon^{N}\left(\sum_{\substack{i, j=1 \\
i \neq j}}^{k} e^{-\left(1+\sigma_{0}\right) \theta_{i j} r / \varepsilon}+e^{-(p+1+2 \sigma)\left(r-r_{f}\right) / \varepsilon}\right)\right) .
\end{aligned}
$$

Suppose $|z|=r=r_{f}+\delta \varepsilon \ln \frac{1}{\varepsilon}$. Then there exist positive constants $C_{1}, C_{2}, C_{3}, C_{4}$ independent of $z$ and $\varepsilon$ such that

$$
\begin{gathered}
\int_{\mathbb{R}^{N}} f(x) U_{\varepsilon, y^{i}}^{p+1} \geq C_{1} \varepsilon^{N} e^{-(p+1+\sigma)\left(r-r_{f}\right) / \varepsilon} \\
=C_{1} \varepsilon^{N+(p+1+\sigma) \delta}, \\
\sum_{\substack{i, j=1 \\
i \neq j}}^{k} \int_{\mathbb{R}^{N}} f(x) U_{\varepsilon, y^{i}}^{p} U_{\varepsilon, y^{j}} \geq C_{2} \varepsilon^{N} \sum_{\substack{i, j=1 \\
i \neq j}}^{k} e^{-\left(1+\frac{\sigma_{0}}{2}\right) \theta_{i j} r / \varepsilon}
\end{gathered}
$$


(see Lemma 3.7 in [10]), and

$$
\varepsilon^{2} \int_{\mathbb{R}^{N}} h(x)\left(\sum_{i=1}^{k} U_{\varepsilon, y^{i}}\right)^{2} \leq C_{3} \varepsilon^{N+2} .
$$

Substituting (4.7), (4.8) and (4.9) into (4.6), we obtain if $\delta>0$ is so fixed such that $(p+1+\sigma) \delta \leq 1$,

$$
J_{\varepsilon}\left(y, \alpha_{\varepsilon, y}, v_{\varepsilon, y}\right) \geq k \varepsilon^{N} A+C_{4} \varepsilon^{N+1}
$$

for some constant $C_{4}>0$, where $z$ satisfies $|z|=r_{f}+\delta \varepsilon \ln \frac{1}{\varepsilon}$ and $y=\left(g z, \ldots, g^{k-1} z, z\right)$.

If $r \gg r_{f}+1$, let $z \in \mathbb{R}^{N}$ be any point such that $|z|=r$ and let $y=\left(g z, \ldots, g^{k-1} z, z\right)$. Then

$$
\begin{aligned}
\sum_{i=1}^{k} \int_{\mathbb{R}^{N}} f(x) U_{\varepsilon, y^{i}}^{p+1} & \leq C_{5} \varepsilon^{N} e^{-(p+1-\sigma)\left(r-r_{f}\right) / \varepsilon}, \\
\sum_{\substack{i, j=1 \\
i \neq j}}^{k} \int_{\mathbb{R}^{N}} f(x) U_{\varepsilon, y^{i}}^{p} U_{\varepsilon, y^{j}} & \leq C_{6} \int_{\mathbb{R}^{N}} f(x) \sum_{i=1}^{k} U_{\varepsilon, y^{i}}^{p+1} \\
& \leq C_{7} \varepsilon^{N} e^{-(p+1-2 \sigma) r / \varepsilon} .
\end{aligned}
$$

On the other hand, for any $\sigma>0$ we have

$$
\begin{aligned}
\int_{\mathbb{R}^{N}} h(x)\left(\sum_{i=1}^{k} U_{\varepsilon, y^{i}}\right)^{2} & \geq \sum_{i=1}^{k} \int_{\mathbb{R}^{N}} h(x) U_{\varepsilon, y^{i}}^{2} \\
& \geq C_{8} \varepsilon^{N} e^{-(2+\sigma)(r+a) / \varepsilon}
\end{aligned}
$$

where $a>0$ is some constant, $C_{8}>0$ if meas $\left\{x \in \mathbb{R}^{N} \mid h(x)>0\right\}>0$, and $C_{8}=0$ otherwise. Similarly,

$$
\int_{\mathbb{R}^{N}} h(x)\left(\sum_{i=1}^{k} U_{\varepsilon, y^{i}}\right)^{2} \leq C_{9} \varepsilon^{N} e^{-(2-\sigma)(r-a) / \varepsilon},
$$

where $C_{9}=0$ if $h(x) \equiv 0, C_{9}>0$ otherwise.

Fixing $r_{0}>0$ large, we get from (4.6) (4.11), (4.12) and (4.13) that for $y_{0}=$ $\left(g z_{0}, \ldots, g^{k-1} z_{0}, z_{0}\right)$ with $\left|z_{0}\right|=r_{0}$

$$
\begin{aligned}
J_{\varepsilon}\left(y, \alpha_{\varepsilon, y}, v_{\varepsilon, y}\right) \leq k \varepsilon^{N} A & -\frac{\varepsilon^{N+2}}{4} C_{8} e^{-(2+\sigma)\left(r_{0}+a\right) / \varepsilon} \\
& -\frac{1}{4} c_{0} \varepsilon^{N} \sum_{\substack{i, j=1 \\
i \neq j}}^{k} e^{-(1+\sigma) \theta_{i j} r_{0} / \varepsilon}
\end{aligned}
$$

( $c_{0}=0$ if $k=1, \sigma>0$ is any number small enough), where we have used the following estimate:

$$
\int_{\mathbb{R}^{N}} U_{\varepsilon, y^{i}}^{p} U_{\varepsilon, y^{j}}=\left(c_{0}+o(1)\right) e^{-\left|y^{i}-y^{j}\right| / \varepsilon}\left(\frac{\varepsilon}{\left|y^{i}-y^{j}\right|}\right)^{\frac{N-1}{2}}
$$

for some $c_{0}>0$, which is obtained in [5] (see also Lemma 3.7 in [10]). 
Applying (4.6), (4.12), (4.14) and (4.16) we have for $y=\left(g z, \ldots, g^{k-1} z, z\right)$ with $|z|=R, R \gg r_{0}$ that for any $\sigma \in(0,1)$,

$$
\begin{aligned}
J_{\varepsilon}\left(y, \alpha_{\varepsilon, y}, v_{\varepsilon, y}\right) \geq k \varepsilon^{N} A & -\varepsilon^{N+2} C_{9} e^{-(2-\sigma)(R-a) / \varepsilon} \\
& -c_{0} \varepsilon^{N} \sum_{\substack{i, j=1 \\
i \neq j}}^{k} e^{-(1-\sigma) \theta_{i j} R / \varepsilon},
\end{aligned}
$$

where we agree that $c_{0}=0$ if $k=1$.

From (4.9), (4.16) and (4.17) we easily get (4.2) if $k=1$, meas $\{x \mid h(x)>0\}>0$ and $k \geq 2$ for $R$ large.

Thus $m_{\varepsilon, \delta, R}$ can only be achieved by some point in $D_{\delta, R}$.

Proof of Theorem 3.1. Let $F\left(y^{1}, \ldots, y^{k}\right)=J_{\varepsilon}\left(y, \alpha_{\varepsilon, y}, v_{\varepsilon, y}\right), L(z)=F\left(z, g z, \ldots, g^{k-1} z\right)$ for $z \in B_{\delta, R}$. We claim that for any permutation $\pi$ of $\mathbb{R}^{k}, j=1, \ldots, k-1, F$ satisfies

$$
\begin{aligned}
F(\pi y) & =F(y), \\
F\left(g^{j} y\right) & =F(y) .
\end{aligned}
$$

Indeed, set $\widehat{\alpha}_{\varepsilon}=\pi \alpha_{\varepsilon, y}, \widehat{v}_{\varepsilon}(x)=v_{\varepsilon, y}(x)$. Then $J_{\varepsilon}\left(\pi y, \widehat{\alpha}_{\varepsilon}, \widehat{v}_{\varepsilon}\right)=J_{\varepsilon}\left(y, \alpha_{\varepsilon, y}, v_{\varepsilon, y}\right)$ and $\left(\pi y, \widehat{\alpha}_{\varepsilon}, \widehat{v}_{\varepsilon}\right)$ satisfies (3.4) and (3.5) with $\widehat{\xi}=\pi \xi, \widehat{\eta}=\pi\left(\eta^{1}, \ldots, \eta^{k}\right)$. It follows from the uniqueness of the $C^{1}$-map obtained in Proposition 3.2 that $\alpha_{\varepsilon, \pi y}=\alpha_{\varepsilon, y}, v_{\varepsilon, \pi y}=v_{\varepsilon, y}$, from which (4.18) can be deduced.

Similarly, setting $\widetilde{\alpha}_{\varepsilon}=\alpha_{\varepsilon, y}, \widetilde{v}_{\varepsilon}(x)=v_{\varepsilon, y}\left(g^{-j} x\right)$, we get

$$
J_{\varepsilon}\left(g^{j} y, \widetilde{\alpha}_{\varepsilon}, \widetilde{v}_{\varepsilon}\right)=J_{\varepsilon}\left(y, \alpha_{\varepsilon, y}, v_{\varepsilon, y}\right)
$$

and $\left(g^{j} y, \widetilde{\alpha}_{\varepsilon}, \widetilde{v}_{\varepsilon}\right)$ satisfies (3.4), (3.5). Hence, as before, $\alpha_{\varepsilon, g^{j} y}=\alpha_{\varepsilon, y}, v_{\varepsilon, g^{j} y}(x)=$ $v_{\varepsilon, y}\left(g^{-j} x\right)$ and (4.19) holds.

On the other hand, setting $\bar{v}_{\varepsilon}(x)=v_{\varepsilon, y}\left(g^{-j} x\right)$ for any $j=1, \ldots, k$, we have $J_{\varepsilon}\left(y, \alpha_{\varepsilon, y}, \bar{v}_{\varepsilon}\right)=J_{\varepsilon}\left(y, \alpha_{\varepsilon, y}, v_{\varepsilon, y}\right)$ and $\left(y, \alpha_{\varepsilon, y}, \bar{v}_{\varepsilon}\right)$ satisfies (3.4), (3.5). Again, by the uniqueness of the $C^{1}$-map, obtained in Proposition [3.2, $v_{\varepsilon, y}\left(g^{-j} x\right)=v_{\varepsilon, y}(x)$. So, $v_{\varepsilon, y}\left(g^{j} x\right)=v_{\varepsilon, y}(x)$ for all $j=1, \ldots, k$.

We claim that if $z \in B_{\delta, R}$ is a critical point of $L(z)$, then

$$
\left.\frac{\partial F\left(y^{1}, \ldots, y^{k}\right)}{\partial y_{\ell}^{i}}\right|_{\left(z, g z, \ldots, g^{k-1} z\right)}=0
$$

for any $i=1, \ldots, k, \ell=1, \ldots, N$. Set

$$
\begin{aligned}
D_{y^{i}} F & =\left(\frac{\partial F}{\partial y_{1}^{i}}, \ldots, \frac{\partial F}{\partial y_{N}^{i}}\right), \\
D L & =\left(\frac{\partial L}{\partial z_{1}}, \ldots, \frac{\partial L}{\partial z_{N}}\right) .
\end{aligned}
$$

Suppose $g z=A z$ for some nonsingular matrix $A: \mathbb{R}^{N} \rightarrow \mathbb{R}^{N}$. Without loss of generality, we take $k=3$ to simplify our presentation:

$$
D L(z)=\left.\sum_{i=1}^{3} D_{y^{i}} F\right|_{\left(z, A z, A^{2} z\right)} A^{i} .
$$


Since $F\left(y^{1}, y^{2}, y^{3}\right)=F\left(A^{-1} y^{1}, A^{-1} y^{2}, A^{-1} y^{3}\right)$ we have

$$
F\left(y^{1}, y^{2}, y^{3}\right)=F\left(A^{-1} y^{2}, A^{-1} y^{1}, A^{-1} y^{3}\right) .
$$

Hence

$$
D_{y^{2}} F\left(y^{1}, y^{2}, y^{3}\right)=\left.D_{y^{1}} F\right|_{\left(A^{-1} y^{2}, A^{-1} y^{1}, A^{-1} y^{3}\right)} A^{-1} \text {. }
$$

In particular

$$
\left.D_{y^{2}} F\right|_{\left(z, A z, A^{2} z\right)} A=\left.D_{y^{1}} F\right|_{\left(z, A^{-1} z, A z\right)} .
$$

But $A^{-1} z=A^{2} z$ and $F\left(y^{1}, y^{2}, y^{3}\right)=F\left(y^{1}, y^{3}, y^{2}\right)$. Therefore

$$
D_{y^{1}} F\left(y^{1}, y^{2}, y^{3}\right)=D_{y^{1}} F\left(y^{1}, y^{3}, y^{2}\right)
$$

and consequently from (4.20) and (4.21),

$$
\begin{aligned}
\left.D_{y^{2}} F\right|_{\left(z, A z, A^{2} z\right)} A & =\left.D_{y^{1}} F\right|_{\left(z, A^{2} z, A z\right)} \\
& =\left.D_{y^{1}} F\right|_{\left(z, A z, A^{2} z\right)} .
\end{aligned}
$$

Similarly, we have

$$
\left.D_{y^{3}} F\right|_{\left(z, A z, A^{2} z\right)} A^{2}=\left.D_{y^{1}} F\right|_{\left(z, A z, A^{2} z\right)^{\prime}} .
$$

From (4.20), (4.23) and (4.24) we deduce that

$$
\left.D_{y^{i}} F\right|_{\left(z, A z, A^{2} z\right)}=0 \text {. }
$$

This proves our claim.

By Lemma 4.1, there exists a point $z_{\varepsilon} \in B_{\delta, R}$ such that $y_{\varepsilon}=\left(g z_{\varepsilon}, \ldots, g^{k-1} z_{\varepsilon}, z_{\varepsilon}\right)$ achieves $m_{\varepsilon, \delta, R}$. Thus, $z_{\varepsilon}$ is a critical point of $L$ and therefore $y_{\varepsilon}$ is a critical point of $J_{\varepsilon}\left(y, \alpha_{\varepsilon, y}, v_{\varepsilon, y}\right)$, that is,

$$
\begin{aligned}
& \frac{\partial J_{\varepsilon}\left(y_{\varepsilon}, \alpha_{\varepsilon, y_{\varepsilon}}, v_{\varepsilon, y_{\varepsilon}}\right)}{\partial y_{\ell}^{2}}+\sum_{j=1}^{k} \frac{\partial J_{\varepsilon}\left(y_{\varepsilon}, \alpha_{\varepsilon, y_{\varepsilon}, v_{\varepsilon, y_{\varepsilon}}}\right)}{\partial \alpha_{j}} \frac{\partial \alpha_{\varepsilon, y_{\varepsilon}, j}}{\partial y_{\ell}^{2}} \\
& +\frac{\partial J_{\varepsilon}\left(y_{\varepsilon}, \alpha_{\varepsilon, y_{\varepsilon}}, v_{\varepsilon, y_{\varepsilon}}\right)}{\partial v}\left(\frac{\partial v_{\varepsilon, y_{\varepsilon}}}{\partial y_{\ell}^{i}}\right)=0,
\end{aligned}
$$

for $i=1, \ldots, k, \ell=1, \ldots, N$. Hence

$$
\begin{aligned}
\frac{\partial J_{\varepsilon}\left(y_{\varepsilon}, \alpha_{\varepsilon, y_{\varepsilon}}, v_{\varepsilon, y_{\varepsilon}}\right)}{\partial y_{\ell}^{i}} & =-\sum_{j=1}^{k} \xi_{j}\left\langle U_{\varepsilon, y_{\varepsilon}^{j}}, \frac{\partial v_{\varepsilon, y_{\varepsilon}}}{\partial y_{\ell}^{i}}\right\rangle_{\varepsilon}-\sum_{j=1}^{k} \sum_{m=1}^{N} \eta_{m}^{j}\left\langle\frac{\partial U_{\varepsilon, y_{\varepsilon}^{j}}^{j}}{\partial y_{m}^{j}}, \frac{\partial v_{\varepsilon, y_{\varepsilon}}}{\partial y_{\ell}^{i}}\right\rangle_{\varepsilon} \\
& =\sum_{m=1}^{N} \eta_{m}^{i}\left\langle\frac{\partial^{2} U_{\varepsilon, y_{\varepsilon}^{i}}}{\partial y_{\ell}^{i} \partial y_{m}^{i}}, v_{\varepsilon, y_{\varepsilon}}\right\rangle_{\varepsilon} .
\end{aligned}
$$

This means that $\left(y_{\varepsilon}, \alpha_{\varepsilon}, y_{\varepsilon}, v_{\varepsilon, y_{\varepsilon}}\right)$ also satisfies (3.6). So $\left(y_{\varepsilon}, \alpha_{\varepsilon, y_{\varepsilon}}, v_{\varepsilon, y_{\varepsilon}}\right)$ is a critical point of $J_{\varepsilon}$ and consequently $u_{\varepsilon}=\sum_{i=1}^{k} \alpha_{\varepsilon, y_{\varepsilon}, i} U_{\varepsilon, y_{\varepsilon}^{i}}+v_{\varepsilon, y_{\varepsilon}}$ is a critical point of $I_{\varepsilon}$ in $H^{1}\left(\mathbb{R}^{N}\right)$. By our proof we know $\alpha_{\varepsilon, y_{\varepsilon}, i}=\alpha_{\varepsilon, y_{\varepsilon}, j}$ for $i, j=1, \ldots, k, v_{\varepsilon, y_{\varepsilon}}\left(g^{j} x\right)=v_{\varepsilon, y_{\varepsilon}}(x)$ for any $j=1, \ldots, k-1$. The fact that $u_{\varepsilon} \geq 0$ is derived from the observation that $u_{\varepsilon}^{-}=\min \left\{u_{\varepsilon}, 0\right\}$ would have lower bound $\left\|u_{\varepsilon}^{-}\right\|_{\varepsilon}^{2} \geq \rho_{0} \varepsilon^{N}$ if $u_{\varepsilon}^{-} \not \equiv 0$, but $\left\|u_{\varepsilon}^{-}\right\|_{\varepsilon}=o(1) \varepsilon^{\frac{N}{2}}$ from our argument of obtaining $v_{\varepsilon, y_{\varepsilon}} \cdot u_{\varepsilon}>0$ follows from the maximum principle for the weak solution. 
Proof of Theorem 2.3. We still keep the notation used above. Conclusions in the sequel can easily be rephrased to derive the results in Theorem 2.3. Let $\left(\alpha_{\varepsilon}, y_{\varepsilon}, v_{\varepsilon}\right) \in M_{\varepsilon, \delta}^{k}$ be a critical point of $J_{\varepsilon}$ as above. By direct calculation, we have

$$
\begin{aligned}
\frac{\partial J_{\varepsilon}\left(\alpha_{\varepsilon}, y_{\varepsilon}, v_{\varepsilon}\right)}{\partial y_{\ell}^{i}}= & -\varepsilon^{2} \int_{\mathbb{R}^{N}} h(x) \sum_{j=1}^{k} U_{\varepsilon, y_{\varepsilon}^{j}} \frac{\partial U_{\varepsilon, y_{\varepsilon}^{i}}}{\partial y_{\ell}^{i}}+\int_{\mathbb{R}^{N}} f(x)\left(\sum_{j=1}^{k} U_{\varepsilon, y_{\varepsilon}^{j}}\right)^{p} \frac{\partial U_{\varepsilon, y_{\varepsilon}^{i}}}{\partial y_{\ell}^{i}} \\
& -p \int_{\mathbb{R}^{N}} \sum_{j \neq i}^{k} U_{\varepsilon, y_{\varepsilon}^{j}}^{p-1} U_{\varepsilon, y_{\varepsilon}^{i}} \frac{\partial U_{\varepsilon, y_{\varepsilon}^{i}}}{\partial y_{\ell}^{i}}+\varepsilon^{N-1} O\left(\sum_{j \neq i}^{k} e^{-(1+\tau)\left|y_{\varepsilon}^{i}-y_{\varepsilon}^{j}\right| / \varepsilon}\right) \\
& +\varepsilon^{N-1} O\left(\varepsilon^{4-N} \int_{\mathbb{R}^{N}} h(x) \sum_{j=1}^{k} U_{\varepsilon, y_{\varepsilon}^{j}}^{2}+\sum_{j=1}^{k} e^{-(1+p+\tau)\left(\left|y_{\varepsilon}^{j}\right|-r_{f}\right) / \varepsilon}\right),
\end{aligned}
$$

where $\tau>0$ is some small constant.

Taking $\varphi=U_{\varepsilon, y_{\varepsilon}^{i}}, \frac{\partial U_{\varepsilon, y_{\varepsilon}^{i}}}{\partial y^{i}}$ in (3.5) respectively we obtain a system of equations for $\xi_{i}, \eta_{\ell}^{i}$ $(i=1, \ldots, k, \ell=1, \ldots, N)$. From the system of equations, using (2.3) we have the following estimate (see the arguments of obtaining (3.35), (3.36) in [12, for example) for $i=1, \ldots, k, m=1, \ldots, N$ :

$$
\begin{aligned}
\eta_{m}^{i}= & \varepsilon^{N+1} O\left(\sum_{j \neq i}^{k} e^{-\left|y_{\varepsilon}^{i}-y_{\varepsilon}^{j}\right| / \varepsilon}\right) \\
& +\varepsilon^{N+1} O\left(\varepsilon^{2-N} \int_{\mathbb{R}^{N}} h(x) \sum_{j=1}^{k} U_{\varepsilon, y_{\varepsilon}^{j}}^{2}+\sum_{j=1}^{k} e^{-(1+p)\left(\left|y_{\varepsilon}^{j}\right|-r_{f}\right) / \varepsilon}\right) .
\end{aligned}
$$

Thus (3.6) is equivalent to

$$
\begin{gathered}
-\varepsilon^{2} \int_{\mathbb{R}^{N}} h(x) \sum_{j=1}^{k} U_{\varepsilon, y_{\varepsilon}^{j}} \frac{\partial U_{\varepsilon, y_{\varepsilon}^{i}}}{\partial y_{\ell}^{i}}+\int_{\mathbb{R}^{N}} f(x)\left(\sum_{j=1}^{k} U_{\varepsilon, y_{\varepsilon}^{j}}\right)^{p} \frac{\partial U_{\varepsilon, y_{\varepsilon}^{i}}}{\partial y_{\ell}^{i}} \\
\quad-p \int_{\mathbb{R}^{N}} \sum_{j \neq i}^{k} U_{\varepsilon, y_{\varepsilon}^{j}}^{p-1} U_{\varepsilon, y_{\varepsilon}^{i}} \frac{\partial U_{\varepsilon, y_{\varepsilon}^{i}}}{\partial y_{\ell}^{i}} \\
=\varepsilon^{N-1} O\left(\sum_{j \neq i}^{k} e^{-(1+\tau)\left|y_{\varepsilon}^{i}-y_{\varepsilon}^{j}\right| / \varepsilon}\right) \\
+\varepsilon^{N-1} O\left(\varepsilon^{4-N} \int_{\mathbb{R}^{N}} h(x) \sum_{j=1}^{k} U_{\varepsilon, y_{\varepsilon}^{j}}^{2}+\sum_{j=1}^{k} e^{-(1+p+\tau)\left(\left|y_{\varepsilon}^{j}\right|-r_{f}\right) / \varepsilon}\right) .
\end{gathered}
$$

(i) Suppose that $h(x)=0$ if $|x| \geq r_{f} ; h(x) \geq h_{0}>0$ if $r_{f}-\bar{\theta} \leq|x|<r_{f}$. If $k=1$, (4.25) becomes

$$
\begin{array}{r}
-\varepsilon^{2} \int_{B_{1}(0)} h(x) U_{\varepsilon, y_{\varepsilon}} \frac{\partial U_{\varepsilon, y_{\varepsilon}}}{\partial y_{\ell}}+\int_{\mathbb{R}^{N}} f(x) U_{\varepsilon, y_{\varepsilon}}^{p} \frac{\partial U_{\varepsilon, y_{\varepsilon}}}{\partial y_{\ell}} \\
=\varepsilon^{N-1} O\left(\varepsilon^{4-N} \int_{\mathbb{R}^{N}} h(x) U_{\varepsilon, y_{\varepsilon}}^{2}+e^{-(1+p+\tau)\left(\left|y_{\varepsilon}\right|-r_{f}\right) / \varepsilon}\right),
\end{array}
$$

Hence, using (2.3) in (4.26) and by direct calculation we get

$$
-\varepsilon^{2}\left(c^{\prime}+o(1)\right) e^{-2\left(\left|y_{\varepsilon}\right|-r_{f}+o(1)\right) / \varepsilon} \frac{\bar{y}_{\varepsilon}-y_{\varepsilon}}{\left|\bar{y}_{\varepsilon}-y_{\varepsilon}\right|}+\left(c^{\prime \prime}+o(1)\right) e^{-(p+1)\left(\left|y_{\varepsilon}\right|-r_{f}+o(1)\right) / \varepsilon} \frac{\bar{y}_{\varepsilon}-y_{\varepsilon}}{\left|\bar{y}_{\varepsilon}-y_{\varepsilon}\right|}=0,
$$


where $\bar{y}_{\varepsilon} \in \partial B_{r_{f}}(0)$ satisfies $\left|\bar{y}_{\varepsilon}-y_{\varepsilon}\right|=\left|y_{\varepsilon}\right|-r_{f}, c^{\prime}$ and $c^{\prime \prime}$ are some positive constants, independent of $\varepsilon, o(1) \rightarrow 0$ as $\varepsilon \rightarrow 0$. Thus we see from the above relation that

$$
-\frac{\left|y_{\varepsilon}\right|-r_{f}}{\varepsilon \ln \varepsilon} \rightarrow \frac{2}{p-1} \quad \text { as } \quad \varepsilon \rightarrow 0 .
$$

For $k \geq 2$, we claim that we still have

$$
-\frac{\left|y_{\varepsilon}^{i}\right|-r_{f}}{\varepsilon \ln \varepsilon} \rightarrow \frac{2}{p-1}, \quad \text { as } \quad \varepsilon \rightarrow 0, \quad i=1, \cdots, k .
$$

In fact, suppose that $\left|y_{\varepsilon}^{i}\right|-r_{f} \geq \eta>0$. In this case, we have

$$
\int_{\mathbb{R}^{N}} f(x) U_{\varepsilon, y_{\varepsilon}^{i}}^{p} \frac{\partial U_{\varepsilon, y_{\varepsilon}^{i}}}{\partial y_{\ell}}=\varepsilon^{N-1} O\left(e^{-(p+1-\sigma)\left(\left|y_{\varepsilon}^{i}\right|-r_{f}\right) / \varepsilon}\right),
$$

for any small $\sigma>0$. Moreover, we have $\left|y_{\varepsilon}^{i}-y_{\varepsilon}^{j}\right| \geq(1+\tau)\left|y_{\varepsilon}^{i}-y_{\varepsilon}^{i-1}\right|$ for some $\tau>0$ if $j \neq i-1, i+1$. Thus (4.25) becomes

$$
\begin{aligned}
& -\varepsilon^{2} \int_{\mathbb{R}^{N}} h(x) U_{\varepsilon, y_{\varepsilon}^{i}} \frac{\partial U_{\varepsilon, y_{\varepsilon}^{i}}}{\partial y_{\ell}^{i}}-p \int_{\mathbb{R}^{N}} \sum_{j=i-1, i+1} U_{\varepsilon, y_{\varepsilon}^{j}}^{p-1} U_{\varepsilon, y_{\varepsilon}^{i}} \frac{\partial U_{\varepsilon, y_{\varepsilon}^{i}}}{\partial y_{\ell}^{i}} \\
= & \varepsilon^{N-1} O\left(\sum_{j \neq i}^{k} e^{-(1+\tau)\left|y_{\varepsilon}^{i}-y_{\varepsilon}^{j}\right| / \varepsilon}\right)+\varepsilon^{N-1} O\left(\sum_{j=1}^{k} e^{-(2+\tau)\left(\left|y_{\varepsilon}^{j}\right|-r_{f}\right) / \varepsilon}\right) \\
& +\varepsilon^{N-1} O\left(\varepsilon^{4-N} \int_{\mathbb{R}^{N}} h(x) \sum_{j=1}^{k} U_{\varepsilon, y_{\varepsilon}^{j}}^{2}+\sum_{j=1}^{k} e^{-(1+p+\tau)\left(\left|y_{\varepsilon}^{j}\right|-r_{f}\right) / \varepsilon}\right) .
\end{aligned}
$$

Thus,

$$
\begin{aligned}
& -\varepsilon^{2}\left(b^{\prime}+o(1)\right) e^{-2\left(\left|y_{\varepsilon}^{i}\right|-r_{f}+o(1)\right) / \varepsilon} \frac{\bar{y}_{\varepsilon}^{i}-y_{\varepsilon}^{i}}{\left|\bar{y}_{\varepsilon}^{i}-y_{\varepsilon}^{i}\right|} \\
& +\left(b^{\prime \prime}+o(1)\right) e^{-\left(\left|y_{\varepsilon}^{i}-y_{\varepsilon}^{i-1}\right|+o(1)\right) / \varepsilon}\left(\frac{y_{\varepsilon}^{i}-y_{\varepsilon}^{i-1}}{\left|y_{\varepsilon}^{i}-y_{\varepsilon}^{i-1}\right|}+\frac{y_{\varepsilon}^{i}-y_{\varepsilon}^{i+1}}{\left|y_{\varepsilon}^{i}-y_{\varepsilon}^{i+1}\right|}\right)=0,
\end{aligned}
$$

where $\bar{y}_{\varepsilon}^{i} \in \partial B_{r_{f}}(0)$ satisfies $\left|\bar{y}_{\varepsilon}^{i}-y_{\varepsilon}^{i}\right|=\left|y_{\varepsilon}^{i}\right|-r_{f}$, and $b^{\prime}>0$ and $b^{\prime \prime}>0$ are some positive constants. Since

$$
-\left\langle\frac{\bar{y}_{\varepsilon}^{i}-y_{\varepsilon}^{i}}{\left|\bar{y}_{\varepsilon}^{i}-y_{\varepsilon}^{i}\right|}, \frac{y_{\varepsilon}^{i}-y_{\varepsilon}^{j}}{\left|y_{\varepsilon}^{i}-y_{\varepsilon}^{j}\right|}\right\rangle \geq \eta>0, \quad i \neq j
$$

we get a contradiction from (4.27). As a result, $\left|y_{\varepsilon}^{i}\right|-r_{f} \rightarrow 0$ as $\varepsilon \rightarrow 0$. Noting that all the interaction terms are exponentially small (of the order $e^{-\theta / \varepsilon}$ for some $\theta>0$ depending on $k$ only), we can take $i=1, \ldots, k$ respectively and argue in exactly the same way as in the case $k=1$ to obtain the desired result.

(ii) Suppose that $k \geq 2$, and $h \equiv 0$. Then for the solutions we constructed, we have $\left|y_{\varepsilon}^{i}-y_{\varepsilon}^{j}\right| \geq(1+\tau)\left|y_{\varepsilon}^{i}-y_{\varepsilon}^{i-1}\right|$ for some $\tau>0$ if $j \neq i-1, i+1$. Thus (4.25) becomes

$$
\begin{aligned}
& \int_{\mathbb{R}^{N}} f(x) U_{\varepsilon, y_{\varepsilon}^{i}}^{p} \frac{\partial U_{\varepsilon, y_{\varepsilon}^{i}}}{\partial y_{\ell}^{i}}-p \int_{\mathbb{R}^{N}} \sum_{j=i-1, i+1} U_{\varepsilon, y_{\varepsilon}^{j}}^{p-1} U_{\varepsilon, y_{\varepsilon}^{i}} \frac{\partial U_{\varepsilon, y_{\varepsilon}^{i}}}{\partial y_{\ell}^{i}} \\
= & \varepsilon^{N-1} O\left(\sum_{j \neq i}^{k} e^{-(1+\tau)\left|y_{\varepsilon}^{i}-y_{\varepsilon}^{j}\right| / \varepsilon}\right)+\varepsilon^{N-1} O\left(\sum_{j=1}^{k} e^{-(1+p+\tau)\left(\left|y_{\varepsilon}^{j}\right|-r_{f}\right) / \varepsilon}\right) .
\end{aligned}
$$


Thus,

$$
\begin{aligned}
& \left(d^{\prime}+o(1)\right) e^{-(p+1)\left(\left|y_{\varepsilon}^{i}\right|-r_{f}+o(1)\right) / \varepsilon} \frac{\bar{y}_{\varepsilon}^{i}-y_{\varepsilon}^{i}}{\left|y_{\varepsilon}^{i}-y_{\varepsilon}^{i}\right|} \\
& +\left(d^{\prime \prime}+o(1)\right) e^{-\left(\left|y_{\varepsilon}^{i}-y_{\varepsilon}^{i-1}\right|+o(1)\right) / \varepsilon}\left(\frac{y_{\varepsilon}^{i}-y_{\varepsilon}^{i-1}}{\left|y_{\varepsilon}^{i}-y_{\varepsilon}^{i-1}\right|}+\frac{y_{\varepsilon}^{i}-y_{\varepsilon}^{i+1}}{\left|y_{\varepsilon}^{i}-y_{\varepsilon}^{i+1}\right|}\right)=0,
\end{aligned}
$$

where $\bar{y}_{\varepsilon}^{i}$ is as before, and $d^{\prime}>0$ and $d^{\prime \prime}>0$ are some positive constants.

Suppose that $y_{\varepsilon} \rightarrow y$ as $\varepsilon \rightarrow 0$. We see from the above relation that

$$
(p+1)\left(\left|y^{i}\right|-r_{f}\right)=\left|y^{i}-y^{i+1}\right| .
$$

But $\left|y^{i}-y^{i+1}\right|=2\left|y^{i}\right| \sin \frac{\pi}{k}$. As a result,

$$
\left|y^{i}\right|=\frac{(p+1) r_{f}}{p+1-2 \sin \frac{\pi}{k}}
$$

\section{REFERENCES}

[1] R.V. Akhmanov, R.V. Khokhlou and A.P. Sukhornkov, Self-focusing, self-defocusing and selfmodulation of laser beams, Laser Handbook, North-Holland, Amsterdam, 1972.

[2] N.N. Akhmediev, Novel class of nonlinear surface waves: Asymmetric modes in a symmetric layered structure, Sov. Phys. JEPT, 56 (1982), 231-247.

[3] A. Ambrosetti, D. Arcoya and J.L. Gámez, Asymmetric bound states of differential equations in nonlinear optics, Rend. Sem. Mat. Univ. Padova, 100 (1998), 231-247. MR1675283 (99m:34103)

[4] D. Arcoya, S. Cingolani and J.L. Gámez, Asymmetric modes in symmetric nonlinear optical waveguides, SIAM J. Math. Anal., 30 (1999), 1391-1400. MR1718307 (2000j:78012)

[5] A. Bahri and Y.Y. Li, On a Min-max procedure for the existence of a positive solution for certain scalar field equations in $\mathbb{R}^{N}$, Revista Math. Iber., 6 (1990), 1-15. MR.1086148 (92b:35054)

[6] A. Bahri and P.L. Lions, On the existence of a positive solution of semilinear elliptic equations in unbounded domains, Ann. Inst. H. Poincaré, Anal. Non Linéaire, 14 (1997), 365-413. MR.1450954 (98k:35047)

[7] H. Berestycki and P.L. Lions, Nonlinear scalar field equations I. Existence of a ground state, Arch. Rat. Mech. Anal., 82 (1983), 313-345. MR0695535 (84h:35054a)

[8] H. Brezis and L. Nirenberg, Positive solutions of nonlinear elliptic equations involving critical Sobolev exponents, Comm. Pure Appl. Math., 36 (1983), 437-477. MR0709644 (84h:35059)

[9] D. Cao, E.N. Dancer, E.S. Noussair and S. Yan, On the existence and profile of multi-peaked solutions to singularly perturbed semilinear Dirichlet problems, Discrete and Continuous Dynamical Systems, 2(1996), 221-236. MR1382508 (96m:35095)

[10] D. Cao and E.S. Noussair, Existence of symmetric multi-peaked solutions to singularly perturbed semilinear elliptic problems, Comm. PDE, 25 (2000), 2185-2232. MR1789925 (2001h:35047)

[11] D. Cao, E.S. Noussair and S. Yan, Solutions with multiple "peaks" for nonlinear elliptic equations, Proc. Royal. Soc. Edinburgh, 129A (1999), 235-264. MR.1686700 (2000a:35052)

[12] D.Cao, E.S. Noussair and S. Yan, Existence and nonexistence of interior-peaked solutions for a nonlinear Neumann problem, Pacific J. Math., 200 (2001), 19-41. MR.1863405 (2002i:35065)

[13] S. Cingolani and J.L. Gámez, Asymmetric positive solutions for a symmetric nonlinear problem in $\mathbb{R}^{n}$, Calc. Var. PDE, 11 (2000), 97-117. MR1777465 (2001f:35121)

[14] S. Cingolani and M. Lazzo, Multiple positive solutions to nonlinear Schrödinger equations with competing potential functions, J. Diff. Equations, 160 (2000), 118-138. MR1734531 (2000j:35079)

[15] V. Coti Zelati and M.J. Esteban, Symmetry breaking and multiple solutions for a Neumann problem in an exterior domain, Proc. Royal Soc. Edinburgh, 116A (1990), 327-339. MR1084737|(91j:35104)

[16] E.N. Dancer and S. Yan, Singularly perturbed elliptic problems in exterior domains, Diff. Integral Equations, 13 (2000), pp. 747-777. MR1750049 (2001c:35022)

[17] M.J. Esteban, Nonsymmetric ground states of symmetric variational problems, Comm. Pure. Appl. Math., 44 (1991), 259-274. MR.1085830 (91m:35073) 
[18] O. John and C. Stuart, Guidance properties of a cylindrical defocusing waveguide, Comm. Math. Univ. Carolinae, 35 (1994), 653-673. MR:1321236 (95m:78023)

[19] M.K. Kwong, Uniqueness of positive solutions of $\Delta u-u+u^{p}=0$ in $\mathbb{R}^{n}$, Arch. Rat. Mech. Anal., 105 (1989), 243-266. MR0969899 (90d:35015)

[20] Y.Y. Li, Existence of multiple solutions of semilinear equations in $\mathbb{R}^{n}$, Progr. Nonlinear Diff. Equations, 4 (1990), 134-159.

[21] J.H. Marburgher, Self-focusing: Theory, Prog. Quant. Electr., 4 (1975), 35-100.

[22] E.S. Noussair and S. Yan, On positive multipeak solutions of a nonlinear elliptic problem, J. London Math. Soc., 62 (2000), 213-227. MR1772182 (2001k:35098)

[23] O. Rey, The role of the Green's function in a nonlinear elliptic equation involving the critical Sobolev exponent, J. Funct. Anal., 89 (1990), 1-52. MR1040954 (91b:35012)

[24] Y.R. Shen, Self-focusing: Experimental, Prog. Quant. Electr., 4 (1975), 1-34.

[25] C. Stuart, Guidance properties of nonlinear planar waveguides, Arch. Rat. Mech. Anal., 125 (1993), 145-200. MR 1245069 (94j:78022)

[26] C. Stuart, Self-trapping of an electromagnetic field and bifurcation from the essential spectrum, Arch. Rat. Mech. Anal., 113 (1990), 65-96. MR1079182 (91j:78010)

[27] X. Wang and B. Zeng, On concentration of positive bound states of nonlinear Schrödinger equations with competing potential functions, SIAM J. Math. Anal., 28 (1997), 633-655. MR1443612 (98e:81032)

[28] Z.-Q. Wang, Existence and symmetry of multi-bump solutions for nonlinear Schrödinger equations, J. Diff. Equations, 159 (1999), 102-137. MR.1726920 (2001h:35176) 